\title{
Scheduling of energy storage
}

\author{
Stan Zachary; Simon Tindemans ${ }^{\dagger}$ Michael Evans $;$ \\ James Cruise ${ }^{\S}$ and David Angeli $\mathbb{I}$
}

November 25, 2020

\begin{abstract}
The increasing reliance on renewable energy generation means that storage may well play a much greater role in the balancing of future electricity systems. We show how heterogeneous stores, differing in capacity and rate constraints, may be optimally, or nearly optimally, scheduled to assist in such balancing, with the aim of minimising the total imbalance (unserved energy) over any given period of time. It further turns out that in many cases the optimal policies are such that the optimal decision at each point in time is independent of the future evolution of the supply-demand balance in the system, so that these policies remain optimal in a stochastic environment.
\end{abstract}

\section{$1 \quad$ Introduction and model}

A future much greater reliance on renewable energy means that there is likely to be corresponding much greater need for storage in order to keep electricity systems balanced - see [16, 12]. The optimal operation of energy storage for such balancing may be considered from the viewpoint of the provider (see [14, 1, 3, 8, 13, 17] and the references therein), or from that of the system operator, who is seeking to schedule given storage resources so as to balance the system as far as possible. The latter problem has only received significant attention relatively recently, and then mostly for the problem of scheduling initially full stores so as to cover periods of continuous energy shortfall - see, e.g., [11] for practical applications in the context of the GB energy system, [9, 15, 19] for dynamic programming and simulation approaches, $[5,7,6,2]$ for underlying theory and [20] for a hybrid approach that uses an analytical discharge policy and a recharging policy based on machine learning.

The present paper considers the problem of optimally scheduling heterogeneous storage resources - characterised by different capacities, input/output rate constraints, and round-trip efficiencies - over extended periods of time in which there are both periods of energy shortfall to be met from storage and periods of energy surplus

\footnotetext{
${ }^{*}$ Heriot-Watt University, Edinburgh, UK. Research supported by EPSRC grant EP/I017054/1

${ }^{\dagger}$ Delft University of Technology, Netherlands and Alan Turing Institute, London, UK

${ }^{\ddagger}$ Imperial College London, UK

${ }^{\S}$ Heriot-Watt University. Research supported by EPSRC grant EP/I017054/1

I Imperial College London, UK and University of Florence, Italy
} 
available to recharge storage. Our main objective is the minimisation of unserved energy demand over any given time horizon, or, in a stochastic environment, the expectation of this - often referred to as expected-energy-not-served (EENS). We work primarily in a deterministic environment. Progress may be made using strong Lagrangian or other closely related techniques - see, e.g., [18] and, for an application of this approach to the present problem, see [2]. However, in a deterministic environment optimal solutions are typically far from unique, and the optimal solution obtained by such techniques as above is often such that at any point in time the optimal decision has some dependence on the future evolution of the external supply-demand balance process. The approach of the present paper does not require the use of such machinery and, for the problems considered in the paper, it turns out that the optimal (or near optimal) policy is generally non-anticipatory, i.e. the optimal decision at each successive time is independent of the evolution of the external supply-demand balance subsequent to that time, so that results continue to hold in a stochastic environment, both for the minimisation of the unserved energy demand and for the minimisation of any quantile of the distribution of the unserved energy demand.

We thus study a given set $S$ of energy stores. At each time $t \in[0, \infty)$, the volume of usable energy (after accounting for any output losses) within each store $i \in S$ is given by $E_{i}(t)$, where the latter is subject to the capacity constraint

$$
0 \leq E_{i}(t) \leq \bar{E}_{i}
$$

A policy for the use of the stores is a specification, for each time $t \geq 0$, of the rate $r_{i}(t)$, at which each store $i \in S$ serves energy at time $t$, where positive values of $r_{i}(t)$ correspond to the store discharging, and negative values of $r_{i}(t)$ correspond to the store charging. Hence, in particular,

$$
E_{i}(t)=E_{i}(0)-\int_{0}^{t} r_{i}(u) d u, \quad \text { for all } t \geq 0 .
$$

For each store $i \in S$, the rates $r_{i}(t)$ are further required to satisfy the power or rate constraints

$$
-P_{i}^{\prime} \leq r_{i}(t) \leq P_{i}, \quad \text { for all } t \geq 0,
$$

for given constants $P_{i}^{\prime} \geq 0$ and $P_{i}>0$. Finally, each store $i \in S$ is assumed to have a round-trip efficiency $0<\eta_{i} \leq 1$, so that, at any time $t$ such that the store is charging (i.e. $r_{i}(t)<0$ ) the rate at which energy must be supplied to the store from some external source is given by $-r_{i}(t) / \eta_{i}$ (recall that the level of energy within a store is measured as that which it may usefully output).

The stores are used to assist in managing some given demand process $(d(t), t \geq 0)$, defined for all times $t \geq 0$, positive values of which correspond to an external energy demand to be met (perhaps partially) from the stores, and negative values of which correspond to an external energy surplus which may be used to recharge the stores. In Section 2 we study the case in which the demand process $(d(t), t \in[0, T])$ is nonnegative over some given time interval $[0, T]$ and is to be served as far as possible over that interval by the stores, subject to the constraint that the latter may only 
discharge for $t \in[0, T]$. In particular this may be appropriate to the situation in which storage is used to cover continuous periods of what would otherwise be energy shortfall (e.g. periods of daily peak demand), but may readily be fully recharged between such periods. We show that there is a policy which minimises the unserved energy demand

$$
\int_{0}^{T} \max \left(d(t)-\sum_{i \in S} r_{i}(t), 0\right) d t,
$$

and in which the rates $r_{i}(t), i \in S$, at each time $t$ depend only on $d(t)$ and the energy in each store at time $t$. This policy therefore continues to be optimal in a stochastic environment. The results in this section gather together - and, in considering arbitrary time intervals, extend - results collectively obtained in $[10,5,7,6,2]$, but are now unified and presented with considerably simpler proofs, laying a necessary foundation for subsequent sections.

In Section 3 we continue to assume a nonnegative demand process over some time interval $[0, T]$, but allow that individual stores may both charge and discharge over that interval, typically corresponding to the situation is which cross-charging between stores is allowed. We show that such cross-charging may occasionally be helpful, but give results which identify common situations in which it is not. In particular, we show that cross-charging cannot be helpful when, as discussed in the preceding paragraph, storage may be fully recharged between periods of external energy shortfall and in which the energy shortfall during such periods is unimodal, increasing to a maximum and thereafter decreasing.

Finally, in Section 4 we consider the general case in which the demand process may be both positive and negative, where negative values have the interpretation given above. We study the situation in which the stores have a common round-trip efficiency, and use earlier results to identify heuristic policies for the (near) optimal management of the storage, and to derive conditions under which they are truly optimal.

\section{Pure discharge model}

In this section we take the demand process $(d(t), t \geq 0)$ to be nonnegative over some time interval of interest. Without loss of generality - by, if necessary, redefining the demand process to be zero outside it - we may take this time interval to consist of the entire positive half-line, so that $d(t) \geq 0$ for all $t \geq 0$. We assume that, throughout this time interval, each store $i \in S$ may only discharge, so that the rate constraint (1.3) is replaced by

$$
0 \leq r_{i}(t) \leq P_{i}, \quad \text { for all } t \geq 0,
$$

and that

$$
\sum_{i \in S} r_{i}(t) \leq d(t), \quad t \geq 0 .
$$

The energy $E_{t}(t)$ in each store $i$ at each time $t \geq 0$ is then as given by (1.2) and is a (weakly) decreasing function of $t$ which we continue to require to satisfy (1.1) 
(though the second inequality in (1.1) plays no part in the analysis of this section). Our objective is to choose rate processes $\left(r_{i}(t), t \geq 0\right)$ for all stores $i \in S$, satisfying the above constraints, with the objective of either satisfying (2.2) with equality for all $t$ in some interval $[0, T]$ where $T$ is as large as possible, or else that of minimising the unserved energy demand, given by (1.4), over any given time interval $[0, T]$ (where we allow also $T=\infty$ ).

Under any given policy for the use of the stores in $S$, the further capabilities of the energy contained within that set at and subsequent to any given time $t \geq 0$ are defined by the rate constraints $P_{i}$ and by the residual stored energies $\left(E_{i}(t), i \in S\right)$ at time $t$. It is helpful to have some efficient way of representing this residual storedenergy configuration. This should be sufficient to characterise the set of residual demand processes $\left(d\left(t^{\prime}\right), t^{\prime} \geq t\right)$ which may be fully served at and subsequent to the time $t$. For any such time $t$, define the (residual) burst-power profile of the storedenergy configuration at that time as the necessarily decreasing function $s^{t}(u)$ of $u$ given by

$$
s^{t}(u)=\sum_{i \in S: E_{i}(t) / P_{i} \geq u} P_{i}
$$

(see also Figure 1). For each store $i \in S$, we refer to the quantity $E_{i}(t) / P_{i}$ as the (residual) discharge-duration of the store $i$ at the time $t$. This is the length of further time for which the store $i$ could supply energy if it did so at its maximum rate. Thus the integral of $s^{t}\left(u^{\prime}\right)$ from 0 to any time $u$ is the maximum amount of further energy which can possibly be supplied by the stores between the times $t$ and $t+u$. Define also the energy-power transform $e_{s}^{t}(p)[7]$ of the burst-power profile at the time $t$ to be given by

$$
e_{s}^{t}(p)=\int_{0}^{\infty} \max \left(0, s^{t}(u)-p\right) d u, \quad p \geq 0 .
$$

This has the interpretation that, for each $t$ and for each $p \geq 0$, the quantity $e_{s}^{t}(p)$ would be the energy supplied above the reference output $p$ if all stores output at their maximum rates for as long as possible (i.e. until empty) subsequent to the time $t$ (again see Figure 1). Note that, for each time $t$, the burst-power profile (function of $u$ ) given by $s^{t}(u)$ is recoverable from the energy-power transform (function of $p$ ) given by $e_{s}^{t}(p)$.

Observe that $e_{s}^{t}(p)$ is a (weakly) decreasing function of both $p$ and $t$. For each $t \geq 0$, the quantity $e_{s}^{t}(0)$ is the total energy in the stores at time $t$, and $e_{s}^{t}(p)$ is a convex, piecewise linear, (weakly) decreasing function of $p$ which is zero for all $p \geq \sum_{i \in S: E_{i}(t)>0} P_{i}$. The quantity $\sum_{i \in S: E_{i}(t)>0} P_{i}$ is also the maximum rate at which demand which may be served by the stored-energy configuration at time $t$, and is of course decreasing in $t$.

For each $T>0$ and for each $t \in[0, T]$, define also the energy-power transform $e_{d}^{t, T}(p)$ of the demand process on the interval $[t, T]$ to be given by

$$
e_{d}^{t, T}(p)=\int_{t}^{T} \max (0, d(u)-p) d u, \quad p \geq 0 .
$$

We allow also $T=\infty$, and write $e_{d}^{t}(p)$ for $e_{d}^{t, \infty}(p)$. The quantity $e_{d}^{t, T}(p)$ has the interpretation that it would be the unserved energy demand over the interval $[t, T]$ 
demand if power were supplied at a constant rate $p$ during that interval. For each $T$ and for each $p \geq 0$, the function $e_{d}^{t, T}(p)$ is (weakly) decreasing in $t$ and, for each $t \geq 0$, the function $e_{d}^{t, T}(p)$ is convex and (weakly) decreasing in $p$ and is zero for all $p \geq \max _{u \geq t} d(u)$.

We shall say that the (residual) stored-energy configuration $\left(E_{i}(t), i \in S\right)$ at any time $t$ is balanced at time $t$ if and only if $E_{i}(t) / P_{i}$ is constant over all stores $i \in S$. If the stored energy configuration is balanced at time $t$, then it may be kept balanced at all subsequent times by always serving energy from each individual store $i \in S$ at a rate which is proportional to $P_{i}$. Thus, in this case and under such a policy, the stores may fully serve any residual demand process $(d(u), u \geq t)$ such that

$$
d(u) \leq \hat{P}, \text { for all } u \geq t, \quad \text { and } \quad \int_{t}^{\infty} d(u) d u \leq \hat{E}(t),
$$

where $\hat{P}=\sum_{i \in S} P_{i}$ and $\hat{E}(t)=\sum_{i \in S} E_{i}(t)$. Since, under any policy, these conditions are clearly also necessary in order that the stores, balanced at time $t$ as above, may fully serve a given residual demand process subsequent to time $t$, it follows that the above policy for the subsequent use of a balanced energy configuration is optimal in terms of its ability to satisfy any requested demand. Indeed the balanced store configuration at time $t$ has the same subsequent energy-serving capability as a single store with the same total energy content $\hat{E}(t)$ and a maximum output rate of $\hat{P}$.

When the residual stored-energy configuration $\left(E_{i}(t), i \in S\right)$ at time $t$ is balanced as above, the corresponding energy-power transform $e_{s}^{t}(p)$ decreases linearly in $p$ from $\hat{E}(t)$ when $p=0$ to zero when $p=\hat{P}$ (and is zero thereafter). The conditions (2.6) on the residual demand process $(d(u), u \geq t)$ are equivalent to $e_{d}^{t}(0) \leq \hat{E}(t)$ and $e_{d}^{t}(\hat{P})=0$, where $e_{d}^{t}(p)$ is the corresponding energy-power transform of that process on $[0, \infty)$. Since the latter is convex, it follows that the residual demand at and subsequent to time $t$ may be completely served if and only if $e_{d}^{t}(p) \leq e_{s}^{t}(p)$ for all $p \geq 0$, and is then served by keeping the residual stored-energy configuration balanced subsequent to time $t$. It is a consequence of Theorem 2.1 below that, under a suitable policy for the use of the stores, this result extends to general stored-energy configurations.

Suppose that, under a given policy for the use of the set of stores $S$, the total rate at which energy is to be served at each time $t \geq 0$ is given by $\bar{d}(t) \leq d(t)$. We shall say that such a policy is a greatest-discharge-duration-first policy if, at each successive time $t$, the rates at which the individual stores $i \in S$ serve energy is given by prioritising the use of the stores in descending order of their discharge-durations $E_{i}(t) / P_{i}$. More exactly, under this policy at each time $t$ the stores are grouped according to their current discharge-durations $E_{i}(t) / P_{i}$ (so that stores belong to the same group if and only if their discharge-durations are equal); sufficient groups are then selected in descending order of their discharge-durations such that, using the stores within each group at their maximum rates (i.e. each store $i$ within a selected group serves energy at a rate $P_{i}$ ), the total rate at which energy is served is the required $\bar{d}(t)$; however, in order to meet precisely the total rate $\bar{d}(t)$, each store in the last group thus selected may only require to serve energy at some fractional 
rate $\lambda P_{i}$ for some constant $\lambda$ such that $0<\lambda \leq 1$. (See Figure 1.) As time $t$ increases, any such greatest-discharge-duration-first policy gradually equalises over stores the discharge-durations $E_{i}(t) / P_{i}$, thus pushing the residual stored energy configuration towards a balanced state as defined above. Additionally, under this policy, once the discharge-durations within any set of stores have become equal they remain equal for all subsequent times. Thus, when groups of stores as defined above coalesce they remain coalesced, and further the (weak) ordering of stores by their discharge-durations remains unchanged over time.

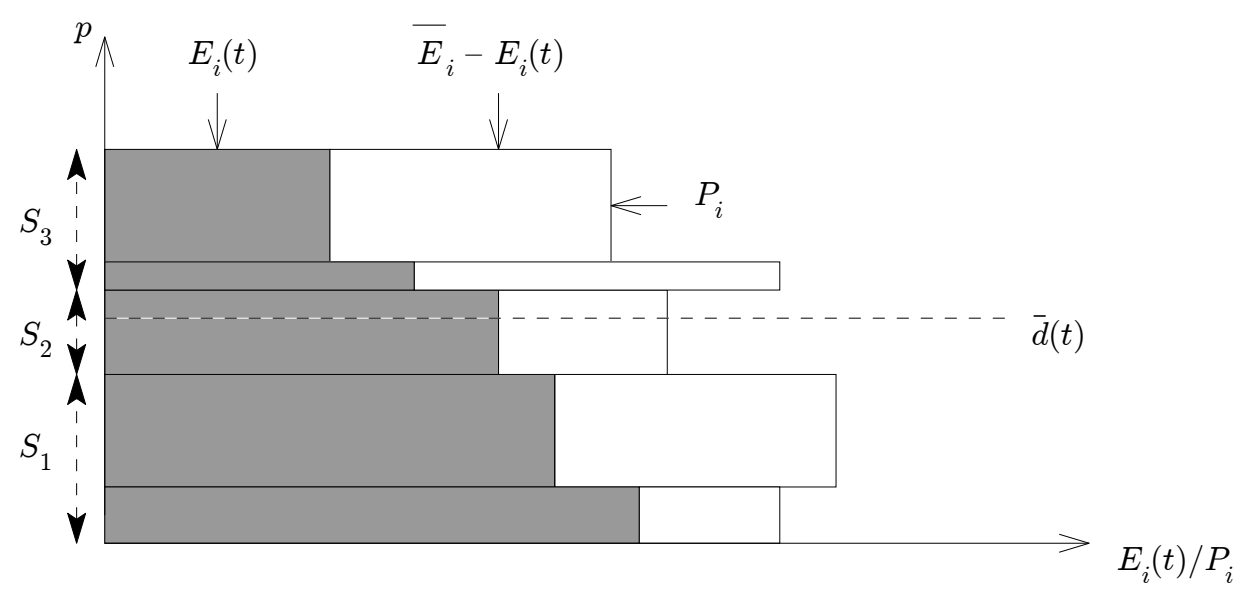

Figure 1: Greatest-discharge-duration-first policy for five stores. The shaded areas correspond to the residual energies $E_{i}(t)$ within the stores at time $t$. Stores are selected in descending order of $E_{i}(t) / P_{i}$. (Thus the total shaded area is the area under the burst-power profile at time $t$, while the shaded area above any level $p$ defines the energy-power transform $e_{s}^{p}(t)$ at time $t$.) When $\bar{d}(t)$ is the energy to be served, $S_{1}, S_{2}$ and $S_{3}$ are the sets of stores which are fully, partially, or not utilised at time $t$.

We shall say that a policy is greedy if, at each successive time $t>0$, it serves as much as possible of the demand $d(t)$ at that time, i.e. if, under this policy,

$$
\sum_{i \in S} r_{i}(t)=\min \left(d(t), \sum_{i \in S: E_{i}(t)>0} P_{i}\right), \quad \text { for all } t \geq 0 .
$$

Note that there is a unique greedy greatest-discharge-duration-first (GGDDF) policy. This policy was independently proposed in [10] in the context of water management, and in $[5,2]$ for the current context of energy storage. A discrete-time algorithm implementing this policy for a piecewise constant demand process is given in [6]. Finally, we note that [20] introduced a closely related discharge policy for the class of constant demand signals $(d(t)=d$, for $t \in[0, T]$ and $d(t)=0$ for $t>T)$, where $d$ (but not $T$ ) is known to the dispatcher in advance. For this limited class of signals, the proposed policy is optimal in the sense that it minimises unserved energy demand. It will be shown below that the GGDDF policy is optimal in the same sense, but for a much broader class of demand signals that includes constant signals as a special case. 
Suppose now that it may not be possible to serve the entire demand process $(d(t), t \geq$ 0 ) and that our objective is the minimisation of the total unserved energy demand (1.4) over some time interval $[0, T]$. Theorem 2.1 below is central to the rest of the paper. It gathers together and provides a unified, economical and accessible treatment of results previously obtained by various authors. In particular the optimality of the GGDDF policy is established in [10, 5, 2], while the implied necessary and sufficient condition for this policy to be able to serve a given demand process is derived by [7, 2], and the expression for the unserved energy demand under this policy is given by [6]. In considering arbitrary time intervals, the theorem also provides a very modest extension of these results.

Theorem 2.1. For the given demand function $(d(t), t \geq 0)$ and the given initial energy configuration $\left(E_{i}(0), i \in S\right)$, the total unserved energy demand (1.4) over any time interval $[0, T]$ is minimised by the GGDDF policy, and this minimum is given by

$$
\max _{p \geq 0}\left(e_{d}^{0, T}(p)-e_{s}^{0}(p)\right) .
$$

Proof. We show first that the use of the GGDDF policy results in unserved energy as given by (2.7). For each $t \geq 0$, let $\bar{d}(t) \leq d(t)$ be the demand actually served at time $t$ under the GGDDF policy. Similarly, for each such $t$, let $E_{i}(t)$ be the energy remaining in each store $i \in S$ at time $t$ under the GGDDF policy and let $e_{s}^{t}(p), p \geq 0$, be the corresponding energy-power transform. Suppose that, at time $t$, stores in $S$ are ranked in descending order of their discharge-durations $E_{i}(t) / P_{i}$ (as is required for the implementation of the GGDDF policy). Let $\pi(t)$ be the set of values of $p$ such that $p=\sum_{i=1}^{j} P_{i}$ for some $j$ such that $E_{j+1}(t) / P_{j+1}<E_{j}(t) / P_{j}$, and include also in the set $\pi(t)$ the values $p=0$ and (if no store is empty) $p=\sum_{i \in S} P_{i}$. (See the example of Figure 2 below.) It follows from the earlier observation that once, under the GGDDF policy, the discharge-durations of any two stores have become equal they remain equal thereafter, that

$$
\pi(u) \supseteq \pi(t), \quad \text { for all } 0 \leq u \leq t .
$$

Further, from (2.4) and from the definition of the GGDDF policy, it is readily checked that, under this policy, at each time $t$ and for all $p \geq 0$, the derivative with respect to $t$ of the energy-power transform $e_{s}^{t}(p)$ of the residual energy configuration satisfies

$$
\frac{d}{d t}\left(e_{s}^{t}(p)\right) \leq \min (0, p-\bar{d}(t))
$$

with equality for all $p \in \pi(t)$. The latter equality is easily seen, and the general result (2.9) follows from the observation that the above derivative varies linearly in $p$ between successive points of the set $\pi(t)$ (again see the example of Figure 2), while the function $\min (0, p-\bar{d}(t))$ is concave in $p$. (Indeed we have equality in (2.9) for all values of $p$ other than for those lying within that interval which is bounded by two consecutive points of the set $\pi(t)$ and within which $\bar{d}(t)$ lies.) Similarly, from (2.5), at each time $t$ and for all $p \geq 0$, the derivative with respect to $t$ of the energy-power transform $e_{d}^{t, T}(p)$ of the residual demand is given by

$$
\frac{d}{d t}\left(e_{s}^{t}(p)\right)=\min (0, p-d(t))
$$


Thus, from (2.9) and (2.10), under the GGDDF policy, at each time $t$ and for all $p \geq 0$,

$$
\frac{d}{d t}\left(e_{s}^{t}(p)-e_{d}^{t, T}(p)\right) \leq \begin{cases}d(t)-\bar{d}(t), & p<\bar{d}(t), \\ d(t)-p, & \bar{d}(t) \leq p \leq d(t), \\ 0, & p>d(t),\end{cases}
$$

with equality for all $p \in \pi(t)$. It now follows from (2.11) that, under the GGDDF policy and for all $p \geq 0$, the unserved energy demand over the interval $[0, T]$ is given by

$$
\begin{aligned}
\int_{0}^{T}(d(t)-\bar{d}(t)) d t & \geq e_{s}^{T}(p)-e_{s}^{0}(p)+e_{d}^{0, T}(p) \\
& \geq e_{d}^{0, T}(p)-e_{s}^{0}(p)
\end{aligned}
$$

where (2.13) follows since necessarily $e_{s}^{T}(p) \geq 0$. It follows that the unserved energy demand over the time interval $[0, T]$ is greater than or equal to the expression given by (2.7). To prove equality, define $\hat{p}=\min \left\{p \geq 0: e_{s}^{T}(p)=0\right\}$. Note that $\hat{p}$ necessarily exists and that $\hat{p} \in \pi(T)$; the latter follows, for example, from the linearity of $e_{s}^{T}(p)$ between adjacent points of $\pi(T)$. Observe that $\hat{p} \leq \bar{d}(t)$ for all $t \in[0, T]$ such that $\bar{d}(t)<d(t)$ (i.e. under the GGDDF policy there is unserved demand at time $t$ ); this follows since, for any such $t$, necessarily $e_{s}^{t}(\bar{d}(t))=0$ and so also $e_{s}^{T}(\bar{d}(t))=0$. Observe also that, by (2.8), $\hat{p} \in \pi(t)$ for all $t \in[0, T]$. It follows from the above two observations, and by using (2.11), that (2.12) holds with equality for $p=\hat{p}$. Since also $e_{s}^{T}(\hat{p})=0$, the relation (2.13) also holds with equality for $p=\hat{p}$. Hence the expression (2.7) also provides an upper bound on the unserved energy demand over the interval $[0, T]$ under the GGDDF policy. (Note that, combining this with the earlier lower bound, it follows (i) that the quantity $e_{d}^{0, T}(p)-e_{s}^{0}(p)$ is maximised for $p=\hat{p}$, and (ii) that when the demand function can be fully served, we have $e_{d}^{0, T}(\hat{p})=e_{s}^{0}(\hat{p})$.) It now follows that, under the GGDDF policy, the unserved energy demand is as given by (2.7).

We now show that any nonnegative demand process $(d(t), t \geq 0)$ which may be completely served over the interval $[0, T]$ by some policy, may be completely served over that interval by the use of the GGDDF policy. To do this, it is sufficient to show that the condition

$$
e_{s}^{0}(p) \geq e_{d}^{0, T}(p) \quad \text { for all } p \geq 0 .
$$

is necessary (as well as sufficient) for the demand process $(d(t), t \geq 0)$ to be capable of being completely served over the interval $[0, T]$. For any $p \geq 0$, let $\tau_{p}$ be the of the set of times $t$ within the interval $[0, T]$ such that $d(t) \geq p$. For the demand process $(d(t), t \geq 0)$ to be completely served over the interval $[0, T]$, it is necessary that

$$
\int_{0}^{m\left(\tau_{p}\right)} s^{0}(u) d u \geq \int_{\tau_{p}} d(u) d u
$$

where $m\left(\tau_{p}\right)$ is the total length of the set of times $\tau_{p}$. That this is so follows since, from (2.3), the integral on the left side of (2.15) is the maximum amount of energy 
which is capable of being served within any set of times of total length $m\left(\tau_{p}\right)$. The relation (2.15) in turn implies that

$$
\int_{0}^{m\left(\tau_{p}\right)} \max \left(0, s^{0}(u)-p\right) d u \geq \int_{\tau_{p}} \max (0, d(u)-p) d u,
$$

since $d(u) \geq p$ on the set $\tau_{p}$. However, this is simply the condition (2.14).

Finally, to complete the proof of the theorem, suppose that the nonnegative demand process $(d(t), t \geq 0)$ is not necessarily completely served over the interval $[0, T]$ by any policy. Consider any policy which minimises the unserved energy demand over the interval $[0, T]$ and let $(\hat{d}(t), t \geq 0)$, with $\hat{d}(t) \leq d(t)$ for all $t$ and $d(t)=0$ for $t>T$, be the process of such demand as is served over $[0, T]$ under that policy. Then, by the result of the preceding paragraph (with $\hat{d}(t)$ replacing $d(t)$ for all $t$ ), the process $(\hat{d}(t), t \geq 0)$ may also be completely served over the interval $[0, T]$ by the use of the GGDDF policy. The GGDDF policy therefore also minimises the unserved energy demand over that interval associated with the original process $(d(t), t \geq 0)$.

Theorem 2.1 has the following immediate corollary, which is fundamental in establishing the energy-power transform of a stored-energy configuration as containing all the information as to which future demand processes may be completely served.

Corollary 2.1. Any given demand process $(d(t), t \geq 0)$ may be completely served over any interval $[0, T]$ (i.e. the unserved energy demand (1.4) is zero) by a given energy configuration with (initial) energy-power transform $\left(e_{s}^{0}(p), p \geq 0\right)$ if and only if $e_{s}^{0}(p) \geq e_{d}^{0, T}(p)$ for all $p \geq 0$. Under this condition the demand process is completely served by the use of the GGDDF policy [7].

Remark 2.1. It follows from Corollary 2.1 and the properties of the function $e_{s}^{0}(p)$ noted earlier that, for a given total volume of stored energy $\sum_{i \in S} E_{i}(0)$ at time 0 , the set of future demand processes which may be completely served is maximised when the stored-energy configuration at time 0 is balanced as defined above, so that the corresponding energy-power transform $e_{s}^{0}(p)$ decreases linearly in $p$.

The GGDDF policy has the important property of being non-anticipatory - as defined in Section 1. It follows that the GGDDF policy remains feasible within a stochastic environment, i.e. when, at each successive time $t$, the demand function $d\left(t^{\prime}\right)$ is known for times $t^{\prime} \leq t$, but not necessarily for $t^{\prime}>t$. Since, by Theorem 2.1. the GGDDF policy thus minimises unserved energy demand for all possible evolutions of the demand function, we have the following further corollary to that theorem.

Corollary 2.2. Suppose that (in a stochastic environment) the objective for the optimal serving of energy is the minimisation of the expectation of the unserved energy demand (1.4), or the minimisation of any quantile of the distribution of the unserved energy demand. Then this objective is achieved by the use of the unique GGDDF policy [6, 2]. 
Corollary 2.3. Suppose that a possibly stochastic demand signal $(d(t), t \geq 0)$ cannot be completely served. In this case, consideration of the truncated signals on $t \in[0, \tilde{T}]$, for $\tilde{T} \geq 0$ shows that the GGDDF policy maximises the time until the storage fleet is first unable to serve all demand [5].

Example 2.1. We illustrate various features of the GGDDF and other policies with a simple example, which is adapted to present needs from one given by [2] and which is reasonably realistic in its dimensioning. Consider a time interval $[0,4]$ and a demand process $(d(t), t \in[0,4])$ given by

$$
d(t)= \begin{cases}200, & 0 \leq t \leq 2 \\ 500, & 2<t \leq 3 \\ 100, & 3<t \leq 4\end{cases}
$$

Consider also 5 stores, initially full, each with the same rate constraint $P_{i}=100$ and with values of $E_{i}$ given by 100, 150,200,200, 250 for $i=1, \ldots, 5$. These stores are to be used to serve as much as possible of the above demand process. If time is measured in hours, power in MW (and so energy in MWh), then this example might correspond to a modest level of shortfall over a four-hour period in a country such as Great Britain, with the stores corresponding to four moderately large batteries. It is readily verified that the GGDDF policy empties all five stores over the time period $[0,3]$, serving all the demand during that time period, and none of the demand during the remaining time period [3,4], thereby leaving (minimised) unserved energy demand of 100. This is as predicted by Theorem 2.1-see Figure 2, which plots the energy-power transforms $e_{s}^{0}(p)$ and $e_{d}^{0}(p)$. Various other policies also empty all the stores and hence minimise unserved energy demand. One such is the GGDDF policy applied to the time-reversed demand process, which serves all the demand except that occurring during the period $[0,0.5]$ (during which none is served). A further such policy is that which uses the greatest-discharge-duration-first policy, not greedily, but rather to serve all demand in excess of a given level $k$. For $k=25$ this policy just serves all the demand in excess of that level, again emptying the stores. However, neither of the above two policies, viewed as algorithms, could be implemented in a stochastic environment, as in each case the decisions to be made at each successive time require a knowledge of the demand process over the entire time period $[0,4]$.

Finally, the heuristic greedy policy studied in [4] arranges the stores in some order and completely prioritises the use of earlier stores (with respect to that order) over later ones. It may be checked that, with respect to the arrangement of the stores in either ascending or descending order of capacity, the suggested policy fails to empty the stores and hence fails to minimise unserved energy demand.

\section{Cross-charging of stores}

We continue to study the situation in which the demand process $(d(t), t \geq 0)$ is nonnegative over some time interval, which we again take, without loss of generality, to be the entire positive half-line, so that $d(t) \geq 0$ for all $t \geq 0$. We now allow that 


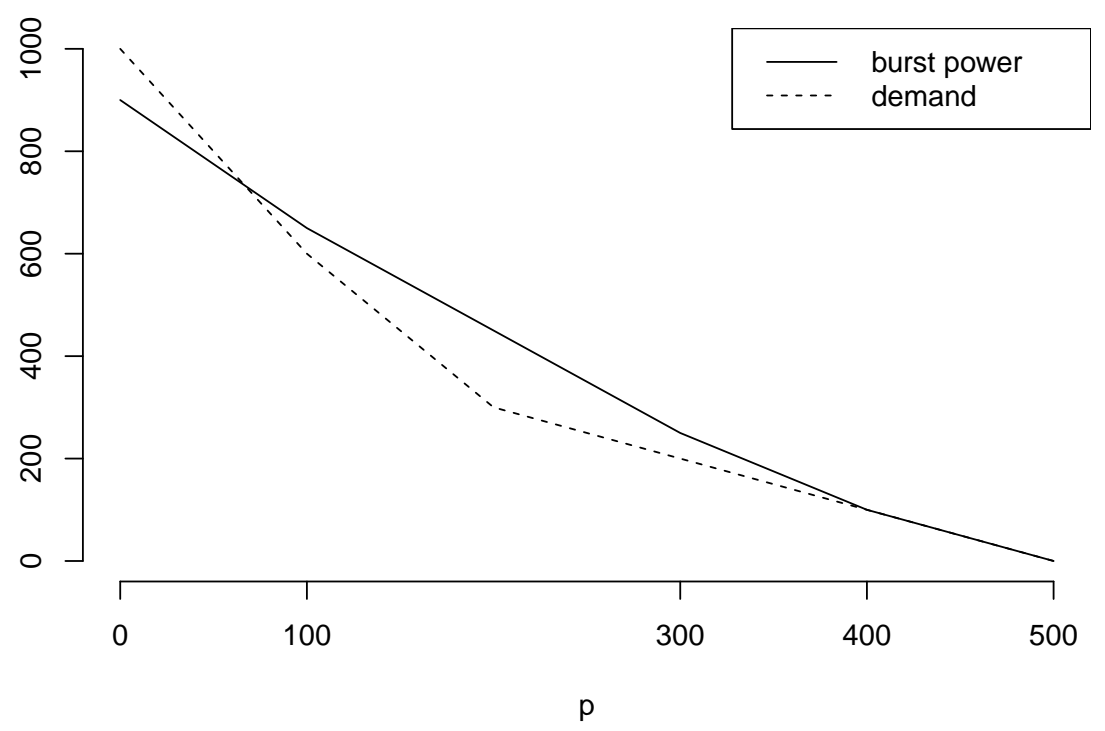

Figure 2: Example 2.1: energy-power transforms $e_{s}^{0}(p)$ and $e_{d}^{0}(p)$ at time 0 of the burst-power profile (of the initial stored-energy configuration) and of the demand process $(d(t), t \geq 0)$. The set $\pi(0)$ defined in the proof of Theorem 2.1 is given by $\{0,100,300,400,500\}$ and is indicated on the horizontal axis. Both $e_{s}^{0}(p)$ and $e_{d}^{0}(p)$ are convex decreasing functions of $p$ and the function $e_{s}^{0}(p)$ is linear between successive points of the set $\pi(0)$. The value of the expression (2.7) is equal to 100 (the maximum being attained at $p=0$ ), which, from Theorem 2.1 is the minimum unserved energy demand over all policies.

stores may be charging as well as discharging, so that, for each $i \in S$, the rates $r_{i}(t)$ satisfy the rate constraints (1.3). However, we require also that the net energy supplied by the stores in $S$ is positive for all $t \geq 0$ and is used to satisfy, again as far as possible in some suitable sense, the demand process $(d(t), t \geq 0)$. Hence we require

$$
0 \leq \sum_{i \in S: r_{i}(t) \geq 0} r_{i}(t)+\sum_{i \in S: r_{i}(t)<0} r_{i}(t) / \eta_{i} \leq d(t), \quad t \geq 0
$$

where, as previously defined, $0<\eta_{i} \leq 1$ is the round-trip efficiency of each store $i \in S$. This corresponds to the situation in which stores may supply energy to each other-which we refer to as cross-charging - but in which no external energy is available for the charging of stores. Our main aim in this section is to show that, while such cross-charging may often assist in serving an external demand, it is also possible to identify circumstances, of considerable importance in practical applications, in which it does not. We give first a simple example in which crosscharging is helpful.

Example 3.1. Consider two stores with capacity and rate constraints (1.1) and (1.3) given by $\left(\bar{E}_{1}, P_{1}\right)=(2,2),\left(\bar{E}_{2}, P_{2}\right)=(4,1)$ and $P_{i}=P_{i}^{\prime}$ for $i=1,2$, and assume further that each store $i$ has a round-trip efficiency $\eta_{i}=1$. We take the demand process $(d(t), t \geq 0)$ to be given by $d(t)=3$ for $t \in[0,1]$ and $t \in[3,4]$ and $d(t)=0$ otherwise. Finally, we assume that the two stores are full at time 0 . Then it is 
straightforward to see the only way in which the demand signal can be completely served for all $t \geq 0$ is to fully empty store 1 and use one unit of energy from store 2 during the time period $[0,1]$, to fully recharge the store 1 from store 2 during the time period $[1,3]$, and then to fully discharge both stores during the time period $[3,4]$.

In the above example there are initial and final periods of high demand, requiring service from both stores, separated by a period of low demand during which the low capacity store may be recharged from the high capacity store. This is the typical situation in which cross-charging may be useful. However, for round-trip efficiencies which are less than one, such cross-charging is inherently wasteful of energy. The following theorem is now fundamental.

Theorem 3.1. Suppose that the demand process $(d(t), t \geq 0)$ is (weakly) decreasing for all $t \geq 0$, and is such that it may be served, possibly with cross-charging and subject to the conditions (3.1), by the given stored-energy configuration. Then it may also be served without cross-charging (i.e. with $r_{i}(t) \geq 0$ for all $i$ and for all $t$ ) by the use of the GGDDF policy.

Proof. We prove the implication of the theorem by proving the contrapositive: if the GGDDF policy (which does not permit cross-charging) cannot serve the demand process $d(t)$, then neither can any other policy, including those that make use of cross-charging.

Clearly, if $d(0)>\sum_{i \in S: E_{i}(0)>0} P_{i}$ (so that there is insufficient power at time $t=0$ ) or if $\int_{0}^{\infty} d(t) \mathrm{d} t>\sum_{i \in S} E_{i}(0)$ (i.e. the total energy in all the stores is insufficient to meet the demand process), then no policy is able to serve the demand process. Hence, we consider only the remaining cases where the GGDDF policy fails to fully serve the demand. In these cases, there must be a first failure time $t=t^{*}$, characterised by the condition

$$
\sum_{i \in S^{*}} P_{i}<d\left(t^{*}\right)
$$

where $S^{*}=\left\{i \in S: E_{i}\left(t^{*}\right)>0\right\}$ is the set of stores that are not empty at time $t^{*}$.

The GGDDF policy has the property that it preserves through time the ordering of discharge-durations $E_{i}(t) / P_{i}$, except for equalisations. Since the stores in $S^{*}$ have the highest values of $E_{i}\left(t^{*}\right) / P_{i}$ at time $t^{*}$ (the other stores being empty at that time), this must also have been the case for all times $t<t^{*}$. Since, further, the demand process $(d(t), t \geq 0)$ is (weakly) decreasing, it now follows from (3.2) that, under the GGDDF policy, all the stores in the set $S^{*}$ have served energy at their maximum rates (i.e. $r_{i}(t)=P_{i}$ for all $i \in S^{*}$ ) for all times $t \in\left[0, t^{*}\right]$. Since also the stores $i \notin S^{*}$ are empty at time $t^{*}$, it now follows that at time $t^{*}$ the GGDDF policy has extracted the maximum possible amount of energy from the entire set of stores $S$ over $t \in\left[0, t^{*}\right]$. Hence, under any policy (with or without cross-charging) which succeeded in serving all demand over the time interval $\left[0, t^{*}\right]$, the stores $i \notin S^{*}$ would also be empty at time $t^{*}$, so that that policy would also fail at that time.

Theorem 3.1 has the following companion result. 
Theorem 3.2. Suppose that the demand function $(d(t), t \in[0, T])$ is (weakly) increasing on $[0, T]$, and that all stores are full at time 0 . Then if $(d(t), t \in[0, T])$ can be served, it can be served without cross-charging (i.e. with $r_{i}(t) \geq 0$ for all $i$ and for all $t$ ), and with at least as much energy remaining in each store at the final time $T$.

Proof. We first consider the case where every store $i \in S$ has a round-trip efficiency $\eta_{i}=1$, so that there is no loss of energy in cross-charging. Here the result can be deduced from Theorem 3.1 by an argument involving time and space reversal. Consider any policy for the use of the stores, possibly involving cross-charging, which serves the given demand process $(d(t), t \in[0, T])$. For each store $i \in S$, let $E_{i}(t)$ be the corresponding level of store $i$ at each time $t$; define a new sequence of storage levels $\left(E_{i}^{*}(t), t \in[0, T]\right)$ on the interval $[0, T]$ for the store $i \in S$ by

$$
E_{i}^{*}(t)=\bar{E}_{i}-E_{i}(T-t), \quad t \in[0, T] .
$$

The set of such sequences over all $i \in S$ corresponds to the use of the stores, with the same input and output rate constraints and again with no loss of energy in crosscharging, to serve fully a demand process $\left(d^{*}(t), t \in[0, T]\right)$ given by $d^{*}(t)=d(T-t)$ for all $t$, with the initial level of each store $i$ being given (from (3.3)) by $E_{i}^{*}(0)=$ $\bar{E}_{i}-E_{i}(T) \leq \bar{E}_{i}$ and with the final level of every store being given by $E_{i}^{*}(T)=0$ (again from (3.3) since, by the hypothesis of the theorem, $E_{i}(0)=\bar{E}_{i}$ for all $i$ ). Further, this pattern of use of the stores involves cross-charging if and only if the use of the original set of sequences $\left(E_{i}(t), t \in[0, T]\right)$, for $i \in S$, to serve the original demand process $(d(t), t \in[0, T])$ similarly involves cross-charging. Since the original demand process is increasing, the demand process $\left(d^{*}(t), t \in[0, T]\right)$ is decreasing and so, by Theorem 3.1, it may also be served fully, without cross-charging, by a modified set of sequences of store levels $\left(\hat{E}_{i}^{*}(t), t \in[0, T]\right), i \in S$, with $\hat{E}_{i}^{*}(0)=E_{i}^{*}(0)$ and $\hat{E}_{i}^{*}(T)=E_{i}^{*}(T)=0$ for all $i$ (this last result following since the sets of processes $\left(E_{i}^{*}(t), t \in[0, T]\right), i \in S$, and $\left(\hat{E}_{i}^{*}(t), t \in[0, T]\right), i \in S$, both start at the same set of levels, serve the same total volume of energy over the period $[0, T]$, and, since the former set of processes fully empties the set of stores, so also must the latter). Finally, transforming back in time and space, it follows that set of sequences of store levels $\left(\hat{E}_{i}(t), t \in[0, T]\right), i \in S$, given by

$$
\hat{E}_{i}(t)=\bar{E}_{i}-\hat{E}_{i}^{*}(T-t), \quad t \in[0, T],
$$

fully serves the original demand process without cross-charging, and that $\hat{E}_{i}(0)=\bar{E}_{i}$ and $\hat{E}_{i}(T)=E_{i}(T)$ for all $i \in S$, as required, and indeed so that in this case the modified process, with cross-charging eliminated, leaves exactly the same volume of energy in each store at the final time $T$.

We now consider the general case $\eta_{i} \leq 1$ for all $i \in S$. Again consider any policy for the use of the stores, possibly involving cross-charging, which serves the given demand process $(d(t), t \in[0, T])$. In particular the rates $r_{i}(t)$ associated with this policy satisfy (3.1) with the second inequality in that expression replaced with equality. As usual, we denote by $E_{i}(t)$ the energy level in each store $i \in S$ at each time $t \geq 0$. Consider also a modified model in which the stores and demand process remain the same, except only that the round-trip efficiencies $\eta_{i}$ are all replaced by 
one. It is clear that we may choose a policy (possibly including cross-charging), i.e. a set of rate functions $\left(\hat{r}_{i}(t), t \geq 0\right), i \in S$, for this modified model in which again all demand is served, i.e.

$$
\sum_{i \in S} \hat{r}_{i}(t)=d(t), \quad t \geq 0
$$

and in which, for each time $t \geq 0$, the corresponding energy levels $\hat{E}_{i}(t)$ in the stores satisfy

$$
\hat{E}_{i}(t) \geq E_{i}(t), \quad i \in S .
$$

To see this observe that we may, inductively over time, choose the rates $\hat{r}_{i}(t)$, and hence the store levels $\hat{E}_{i}(t)$ as follows: at each time $t$, for those $i$ such that $r_{i}(t)<0$, set $\hat{r}_{i}(t)=r_{i}(t)$ unless $E_{i}(t)=\bar{E}_{i}$ (store $i$ is full) in which case set $\hat{r}_{i}(t)=0$; similarly, at each time $t$, for those $i$ such that $r_{i}(t) \geq 0$, choose $0 \leq \hat{r}_{i}(t) \leq r_{i}(t)$ and such that equation (3.4) is satisfied for the modified model. Arguing inductively, it is easy to see that, at each successive point in time, this modified model preserves all rate and capacity constraints together with the relation (3.5). (Informally we might think of the modified model as corresponding to the idea that the same external demand is notionally served at the same rates from the same stores as in the unmodified model, while the perfect round-trip efficiencies of the stores in the modified model enable cross-charging to be used to ensure that, at all times, the relation (3.4) is satisfied and all store levels are greater than or equal to their levels in the unmodified model.) By the result of the theorem already proved for the case of perfect round trip efficiency, the above policy may now be further modified so as to serve the same demand process while eliminating cross-charging and leaving the final volume of energy (at time $T$ ) in each store $i \in S$ unchanged by this elimination. Since no cross-charging is now taking place, this further modified policy now also serves the demand in the original system with $\eta_{i} \leq 1$ for all $i$, again with the same final volume of energy in each store at time $T$, and this volume is therefore greater than or equal to that which was present in the original model when cross-charging was used.

The need, in Theorem 3.2, for some condition such as the requirement that all stores are full at time 0 is shown by Example 3.2 below.

Example 3.2. Consider two stores with capacity and rate constraints (1.1) and (1.3) given by $\left(\bar{E}_{1}, P_{1}\right)=(2,1),\left(\bar{E}_{2}, P_{2}\right)=(1,1)$, with $P_{i}=P_{i}^{\prime}$ for $i=1,2$, and with round-trip efficiencies $\eta_{i}=1$ for $i=1,2$. Consider a time horizon $T=2$ and let the demand process $(d(t), t \in[0,2])$ to be given by $d(t)=0$ for $t \in[0,1]$ and $d(t)=2$ for $t \in[1,2]$. Finally, assume that, at time 0 , store 1 is full while store 2 is empty. Then it is easy to see the only way in which the demand signal can be completely served for all $t \in[0,2]$ is to fully charge store 2 from store 1 during the time period $[0,1]$ and to then fully discharge both stores during the time period $[1,2]$. Hence in this case the need for cross-charging cannot be dispensed with.

Theorems 3.1 and 3.2 have the following corollary.

Corollary 3.1. Suppose that the demand process $(d(t), t \in[0, T])$ is (weakly) increasing on $\left[0, T^{\prime}\right]$ and (weakly) decreasing on $\left[T^{\prime}, T\right]$ for some $0 \leq T^{\prime} \leq T$, and that 
all stores are full at time 0 . Then if $(d(t), t \in[0, T])$ can be served, it can be served without cross-charging, and by the use of the GGDDF policy.

Proof. This is an application of Theorem 3.2 for the period $\left[0, T^{\prime}\right]$-including the result that any cross-charging may be eliminated in this period without reducing the volume of energy in each store at time $T^{\prime}$-followed by the use of Theorem 3.1 for the period $\left[T^{\prime}, T\right]$ (since clearly any increase in the volume of energy in each store at the time $T^{\prime}$ due to the elimination of cross-charging in $\left[0, T^{\prime}\right]$ means that we may continue to serve the given demand process on the interval $\left[T^{\prime}, T\right]$. Finally, since no cross-charging is necessary, it follows from Theorem 2.1 that the demand $(d(t), t \in[0, T])$ can be served by the use of the GGDDF policy.

The following further corollary is an immediate application of Theorem 2.1 and Corollary 3.1.

Corollary 3.2. Under the conditions of Corollary 3.1, and in a possibly stochastic environment, the minimisation of the expectation of the unserved energy demand (1.4), or the minimisation of any quantile of the distribution of the unserved energy demand, is achieved by the use of the GGDDF policy.

An important application of the above result is to the frequently occurring case where stores may be fully recharged overnight, and there is a single period of shortfall during the day which is unimodal in the sense that it is monotonic increasing and then decreasing as in the statement of Corollary 3.1. One may assume that there is no surplus energy available for charging any of the stores during this period. Then, in a possibly stochastic environment, the expectation of the unserved energy demand (1.4) is minimised by the use of the unique GGDDF policy.

Finally in this section, and for completeness, Theorem 3.3 below gives a useful variation of Theorem 3.2. As should be clear from the statement of Theorem 3.2 itself, the condition of that theorem that "all stores are full at time 0" may be relaxed subject to the additional restriction of some minimum level on the demand function. Theorem 3.3 makes this idea precise. It does of course also have corollaries analogous to Corollaries 3.1 and 3.2 above.

Theorem 3.3. Consider any initial stored-energy configuration $\left(E_{i}(0), i \in S\right)$. Let $S^{\prime}=\left\{i \in S: E_{i}(0)<\bar{E}_{i}\right\}$ and define $u_{0}=\min _{i \in S^{\prime}}\left(E_{i}(0) / P_{i}\right)$, with $u_{0}=\infty$ if all stores in $S$ are initially full. Let $S_{1}$ be the set of stores $i$ such that $\bar{E}_{i} / P_{i} \leq u_{0}$. (Note that from the definition of $u_{0}$ all the stores in the set $S_{1}$ are necessarily full.) Define also $S_{2}=S \backslash S_{1}$ and let $k=\sum_{i \in S_{2}} P_{i}$. Suppose that the demand function $(d(t), t \in[0, T])$ is (weakly) increasing, further satisfies $d(0) \geq k$, and may be served possibly with the use of cross-charging. Then it may also be served without crosscharging.

Proof. Consider a modified set of store capacities $\left(\hat{E}_{i}, i \in S\right)$ given by

$$
\hat{E}_{i}= \begin{cases}\bar{E}_{i}, & i \in S_{1}, \\ E_{i}(0)+T^{\prime} P_{i}, & i \in S_{2},\end{cases}
$$


where the constant $T^{\prime}=\max _{i \in S_{2}}\left(\bar{E}_{i}-E_{i}(0)\right) / P_{i}$. Observe that these modified store capacities are all at least as great as the original capacities. Let the demand function $(d(t), t \in[0, T])$ be as in the statement of the theorem. Extend the time interval $[0, T]$ to $\left[-T^{\prime}, T\right]$ and consider the demand function $\left(\hat{d}(t), t \in\left[-T^{\prime}, T\right]\right)$ on this latter interval given by

$$
\hat{d}(t)= \begin{cases}k, & t \in\left[-T^{\prime}, 0\right] \\ d(t) & t \in[0, T]\end{cases}
$$

If the modified set of stores are considered to be full at time $-T^{\prime}$ then their energy content is sufficient to serve the demand function $\left(\hat{d}(t), t \in\left[-T^{\prime}, T\right]\right)$, again possibly with the use of cross-charging. To see this, observe that the stores in the set $S_{2}$ may be utilised at their full rates to directly serve the demand $\hat{d}(t)=k$ on the interval $\left[-T^{\prime}, 0\right]$ (as would be the case with the use of the GGDDF algorithm on that interval); at time 0 the remaining energy in each store $i$ is then the original energy content $E_{i}(0)$ of that store in the initial energy configuration of the theorem; the demand $\hat{d}(t)=d(t)$ on the remaining interval $\left[-T^{\prime}, T\right]$ may now be served as in the statement of the theorem (possibly with cross-charging, since the modified stores are all at least as large as the original stores). Since the demand function $(\hat{d}(t), t \in$ $\left.\left[-T^{\prime}, T\right]\right)$ is, by construction, increasing on $\left[-T^{\prime}, T\right]$, it follows from Theorem 3.2 that this demand function may also be served by the modified stores without crosscharging, and in particular by the use of the GGDDF algorithm. Since the residual energy content of these stores at time 0 is again simply the original energy content of the original stores at time 0 , the conclusion of the theorem now follows.

The need, in Theorem 3.3, for some condition such as the requirement that the demand function $(d(t), t \geq 0)$ has some minimum level at time 0 is again shown by the earlier Example 3.2. The issue here is essentially the same as in Theorem 3.2: informally, for a weakly increasing demand function, it is the combination of an initially sufficiently low level of demand and spare capacity in the stores at time 0 , which enables cross-charging to assist in fully serving the demand function where, in the absence of such cross-charging, this might not be possible.

\section{Charging and discharging}

We now allow that, for every time $t \geq 0$, both the demand $d(t)$ and the rates $r_{i}(t)$, $i \in S$, may be arbitrary (in particular may take either sign) subject to the constraints given by (1.1)-(1.3) and the condition

$$
\min (d(t), 0) \leq \sum_{i \in S: r_{i}(t) \geq 0} r_{i}(t)+\frac{1}{\eta} \sum_{i \in S: r_{i}(t)<0} r_{i}(t) \leq \max (d(t), 0), \quad t \geq 0
$$

where, throughout this section, we assume that all stores $i \in S$ have the same round-trip efficiency $\eta_{i}=\eta$. (For some discussion of the case where the stores have different round-trip efficiencies, see below and also Section 5.) Thus, when the demand $d(t) \geq 0$ the stores collectively serve energy (possibly with cross-charging) to assist in meeting that demand and when $d(t)<0$, corresponding to a surplus of 
energy external to the stores, some of that surplus may be supplied to the stores (again possibly with cross-charging). Our objective continues to be to manage the stores so as to minimise, over those times $t$ such that $d(t) \geq 0$, the long-term unserved energy demand given by (1.4), or, in a stochastic environment, the expectation of this quantity.

For any continuous period of time $\left[T, T^{\prime}\right]$ over which $d(t) \leq 0$ (energy may be supplied to charge stores), there is now a theory which is fully analogous to that developed in Section 2 for discharging stores. In particular we may define the (residual) chargeduration of any store $i \in S$ at any time $t$ as $\left(E_{i}-E_{i}(t)\right) / P_{i}^{\prime}$ (the time that would be required to fully charge the store if this was done at the maximum possible rate). Further the GGDDF policy for discharging-only which is optimal in the sense of Theorem 2.1 is replaced by the analogous greedy greatest-charge-duration-first (GGCDF) policy, which, in the absence of cross-charging, is similarly optimal for attempting to accept as much charge as possible over the interval $\left[T, T^{\prime}\right]$. (Note that this would no longer be true in the absence of a common round-trip efficiency for the stores. While one might, in this case, continue to formulate a result analogous to Theorem 2.1 by redefining store capacities and input rates in terms of external energy input rather than output, the results of the present section would not then continue to hold.)

In many circumstances a reasonable heuristic policy for the management of the stores is given by the use of the GGDDF policy to serve as much of the demand process as possible during periods when that process is positive, and the use of the GGCDF policy to charge the stores as rapidly as possible during periods when the demand process is negative. This policy again has the attractive property, discussed in Section 2, of being non-anticipatory, so that it continues to be fully implementable in a stochastic environment. As in Section 3, this policy may be expected to work particularly well when continuous periods of storage discharge are separated by lengthy periods providing ample time for recharging. However, in general, it need not always be optimal for the minimisation of long-term unserved energy. The reason for this is that the GGDDF policy attempts to equalise as quickly as possible the discharge-durations of the stores, while the GGCDF policy attempts to equalise as quickly as possible the corresponding charge-duration times, and these two objectives in general conflict with each other. However, we do have the following theorem.

Theorem 4.1. Suppose that a set of stores $S$ is such that $\bar{E}_{i} / P_{i}$ is the same for all $i \in S$, that $P_{i}^{\prime}=\alpha P_{i}$ for all $i \in S$ for some $\alpha>0$, and that the stores in $S$ have a common round-trip efficiency $\eta$. Suppose also that $E_{i}(0) / P_{i}$ is the same for all $i \in S$. Then an optimal policy for the minimisation of the expectation of the unserved energy demand (1.4) over any subsequent time period $[0, T]$ is given by the use of the GGDDF policy at those times $t$ such that $d(t)$ is positive, and the use of the GGCDF policy at those times $t$ such that $d(t)$ is negative.

Proof. Under the conditions of the theorem, at any time $t$, balance with respect to charging $\left(E_{i}(t) / P_{i}\right.$ constant over $\left.i \in S\right)$ is equivalent to balance with respect to discharging $\left(\left(\bar{E}_{i}-E_{i}(t)\right) / P_{i}^{\prime}\right.$ constant over $\left.i \in S\right)$. The initial stored-energy config- 
uration is balanced, and so, since the stores have a common round-trip efficiency $\eta$, the GGDDF/GGCDF policy maintains a balanced stored-energy configuration for all $t \geq 0$. It now follows from the results of Section 2 - see in particular Remark 2.1that any demand process $(d(t), t \geq 0)$ which may be completely managed (i.e. completely served at times $t$ such that $d(t) \geq 0$ and completely utilised for charging at times $t$ such that $d(t)<0)$ under some policy may similarly be completely managed by the use of the GGDDF/GGCDF policy. The conclusion of the theorem now follows as for Corollary 2.2.

Remark 4.1. The essence of Theorem 4.1 is that, under the conditions on device parameters given by the theorem, once stores become balanced with respect to their initial energy configurations, they remain so thereafter and may then be used with the same flexibility as a single large store. It follows as in Section 2 that the GGDDF/GGCDF policy drives any initial energy configuration towards such a balanced state. However, cross-charging may speed such convergence.

\section{Concluding remarks}

The present paper has considered the optimal or near optimal scheduling of heterogeneous storage resources for the ongoing balancing of electricity supply and demand. The results, which hold in both deterministic and stochastic environments, are particularly applicable when storage is used to cover periods of energy shortfall, such as periods of daily peak demand, and may be completely or mostly recharged between such periods. However, in future years storage may also be used to balance electricity systems over much longer timescales, such as between summer and winter. The simultaneous existence of multiple timescales, together with the physical characteristics of such storage types as are available, is likely to lead to a very heterogeneous storage fleet, in which, in particular, round-trip efficiencies vary considerably. The optimal dimensioning and control of such storage presents significant further research challenges.

Another topic of ongoing research is to extend the storage dispatch methodology beyond a scenario with a central dispatcher and develop, for example, hierarchical schemes for aggregation and disaggregation of energy units.

\section{Acknowledgements}

The authors are grateful to the Isaac Newton Institute for Mathematical Sciences in Cambridge and to National Grid plc for their support for the programme in which much of the current work was carried out. They also thank Andy Philpott for some helpful literature discussion. Finally, the authors are especially grateful to the reviewers for their most careful and thoughtful readings of the paper and helpful comments and suggestions. 


\section{References}

[1] J. R. Cruise and S. Zachary. The optimal control of storage for arbitrage and buffering, with energy applications. In Renewable Energy: Forecasting and Risk Management, pages 209-227. Springer, 2018.

[2] J. R. Cruise and S. Zachary. Optimal scheduling of energy storage resources. http://arxiv.org/abs/1808.05901, 2018.

[3] P. Denholm, E. Ela, B. Kirby, and M. R. Milligan. The role of energy storage with renewable electricity generation. Technical Report NREL/TP-6A2-47187, National Renewable Energy Laboratory, 2010.

[4] G. Edwards, S. Sheehy, C. J. Dent, and M. C. M. Troffaes. Assessing the contribution of nightly rechargeable grid-scale storage to generation capacity adequacy. Sustainable Energy, Grids and Networks, 12:69-81, 2017.

[5] M. Evans, S. H. Tindemans, and D. Angeli. Robustly maximal utilisation of energy-constrained distributed resources. In 2018 Power Systems Computation Conference (PSCC), pages 1-7, 2018.

[6] M. P. Evans, S. H. Tindemans, and D. Angeli. Minimizing unserved energy using heterogeneous storage units. IEEE Transactions on Power Systems, 34(5):3647$3656,2019$.

[7] M. P. Evans, S. H. Tindemans, and D. Angeli. A graphical measure of aggregate flexibility for energy-constrained distributed resources. IEEE Transactions on Smart Grid, 11(1):106-117, 2020.

[8] N. G. Gast, D. C. Tomozei, and J-Y. Le Boudec. Optimal storage policies with wind forecast uncertainties. In Greenmetrics 2012. Imperial College, London, UK, 2012.

[9] A. S. M. Khan, R. A. Verzijlbergh, O. C. Sakinci, and L. J. De Vries. How do demand response and electrical energy storage affect (the need for) a capacity market? Applied Energy, 214:39-62, 2018.

[10] Peter Nash and Richard Weber. A simple optimizing model for reservoir control. Technical report, University of Cambridge, Cambridge, 1978.

[11] National Grid plc. Electricity Capacity Report. https://www.emrdeliverybody.com/Capacity\%20Markets\%20Document\%20Library/Electricity\%20Ca 2018. Accessed 22 March 2020.

[12] D. M. Newbery, G. Strbac, D. Pudjianto, P. Noël, Booz \& Co., and LeighFisher. Benefits of an integrated european energy market: Final report for DG ENER. https://ec.europa.eu/energy/sites/ener/files/documents/20130902_energy_integration_benefits.pdf, 2013.

[13] D. Pudjianto, M. Aunedi, P. Djapic, and G. Strbac. Whole-systems assessment of the value of energy storage in low-carbon electricity systems. IEEE Transactions on Smart Grid, 5:1098-1109, 2014. 
[14] R. Sioshansi, P. Denholm, T. Jenkin, and J. Weiss. Estimating the value of electricity storage in PJM: Arbitrage and some welfare effects. Energy Economics, 31(2):269-277, 2009.

[15] R. Sioshansi, S. H. Madaeni, and P. Denholm. A dynamic programming approach to estimate the capacity value of energy storage. IEEE Transactions on Power Systems, 29(1):395-403, 2014.

[16] G. Strbac, M. Aunedi, D. Pudjianto, P. Djapic, F. Teng, A. Sturt, D. Jackravut, R. Sansom, V. Yufit, and N. Brandon. Strategic Assessment of the Role and Value of Energy Storage Systems in the UK Low Carbon Energy Future. https://www.carbontrust.com/resources/energy-storage-systemsin-the-uk-low-carbon-energy-future-strategic-assessment, 2012. Accessed 21 June 2020.

[17] T. Weitzel and C. H. Glock. Energy management for stationary electric energy storage systems: A systematic literature review. European Journal of Operational Research, 264(2):582-606, 2018.

[18] P. Whittle. Optimization Under Constraints: Theory and Applications of Nonlinear Programming. Wiley, 1971.

[19] Y. Zhou, P. Mancarella, and J. Mutale. Modelling and assessment of the contribution of demand response and electrical energy storage to adequacy of supply. Sustainable Energy, Grids and Networks, 3:12-23, 2015.

[20] D. Zhu and Y. A. Zhang. Optimal coordinated control of multiple battery energy storage systems for primary frequency regulation. IEEE Transactions on Power Systems, 34(1):555-565, 2019. 